\title{
Calculating Endomorphism Valued Cohomology: Singlet Spectrum in Superstring Models
}

\author{
Jacques Distler ${ }^{1 \cdot \star}$, Brian R. Greene $e^{2, \star \star \star \star \star \star}$, Kelley Kirklin ${ }^{2 \cdot \star \star \star \star \star \star \star \star \star ~}$ \\ and Paul Miron ${ }^{3}$ \\ ${ }^{1}$ F. R. Newman Laboratory of Nuclear Science, Cornell University, Ithaca, NY 14853, USA \\ ${ }^{2}$ Lyman Laboratory of Physics, Harvard University, Cambridge, MA 02138, USA \\ ${ }^{3}$ Swaps Department, County NatWest, Drapers Gardens, London, U.K.
}

\begin{abstract}
We present a computational strategy based on exact sequences for determining the dimension of endomorphism valued cohomology groups for complete intersections in complex projective space. This cohomology group gives rise to part of the gauge singlet spectrum in superstring compactifications. We establish the underlying justification for the known deformation theoretical algorithm, and by comparison with the exact sequence method, indicate its limitations.
\end{abstract}

\section{Introduction}

Any attempt to extract phenomenological predictions from the compactified superstring requires, as the most basic ingredient, an understanding of the low energy particle content. As is by now quite familiar, for smooth manifold compactification this corresponds to an understanding of the cohomology of the compactified manifold as well as that of certain holomorphic vector bundles constructed upon it. To be concrete, we specialize to the $E_{8} \times E_{8}$ Heterotic string [1] compactified on a Calabi-Yau manifold $K$ (a complex threefold of $S U(3)$ holonomy). Although there are other possibilities (for example orbifolds [2]), Calabi-Yau compactification has so far yielded the most realistic superstring phenomenology [3]. As described in $[3,4]$ the massless particle content of such a theory is given by bundle valued $\bar{\partial}$ harmonic forms $H^{*}\left(K, B_{i}\right)$, where the $B_{i}$ are various vector bundles associated to the vacuum gauge bundle $V_{1}$ to which (half of) the left moving world sheet fermions couple [1] (more precisely, vector bundles associated to the principle bundle $\mathscr{V}_{1}$ associated to $V_{1}$ ). The latter arise from the representations of $S$, the structure group of $\mathscr{V}_{1}$ which appear in the decomposition

\footnotetext{
* Work supported in part by NSF contract PHY-82-09011

** Work supported in part by an NSF Postdoctoral Fellowship and DOE contract DE-FG0288ER25065

$\star \star \star$ Work supported in part by NSF contract PHY-82-15249

$\star \star \star \star$ Address after 10 September: Derivative Products Group, Bankers Trust Company, New York, NY, USA
} 
of the 248 of $E_{8}$ under $S \times G$, with $G$ the maximal subgroup of $E_{8}$ commuting with $S$ [5]. In the most familiar case of "embedding the spin connection in the gauge group," we take $V_{1}=T$, where $T$ is the tangent bundle of $K$, thus giving $S=S U(3)$ and $G=E_{6}$. This gives rise to the decomposition

$$
248=(78,1)+(27,3)+(\overline{2} \overline{7}, \overline{3}),+(1,8),
$$

where the numbers in parentheses are the $E_{6}$ and $S U(3)$ representations. The $(78,1)$ are the $E_{6}$ gauge bosons, the $(27,3)$ and $(\overline{2} \overline{7}, \overline{3})$ are matter multiplets in the fundamental representation of $E_{6}$, which occur with multiplicities $\operatorname{dim} H^{1}(K, T)$ $=\operatorname{dim} H^{2,1}(K)$ and $\operatorname{dim} H^{1}\left(K, T^{*}\right)=\operatorname{dim} H^{1,1}(K)$ respectively. These numbers have been computed and used in model building for certain Calabi-Yau manifolds $[3,6,7]$, and recently they have been tabulated for all Calabi-Yau manifolds of the complete intersection type [8]. In this letter we address the problem of the last term in Eq. (1.1), that is a number of $E_{6}$ singlets in the low energy theory, which as we see is given by $\operatorname{dim} H^{1}(K$, End $T)$, where End $T$ represents the bundle of endomorphisms of the tangent bundle. These fields can play an important role in low energy phenomenology both through Yukawa couplings to ordinary matter, nonrenormalizable interactions which can affect flat directions in the superpotential, and have been used in supersymmetry breaking schemes. It is thus important to know at the very least how many such fields a given compactification yields.

\section{Computing $H^{1}(K$, End $T)$}

From previous work on computing $H^{1}(K, T)[9,10]$ we are familiar with two basic approaches to the computation of cohomology: deformation theory and exact sequences. In fact these two approaches are closely related, with exact sequences providing the justification for deformation theory. More precisely, the study of exact sequences yields precise conditions under which deformation theoretical arguments are valid. When these conditions are not met, the only recourse is to perform the full exact sequence computation. Thus we see that the exact sequence computation lies at the heart of the issue. As, such, we shall first present an exact sequence procedure for the computation of the dimension of $H^{1}(K$, End $T)$ and then show how a deformation theoretic interpretation of this computational strategy is sometimes valid.

Before specific computations, it is worthwhile for us to present the general strategy of the computation. For ease of presentation and explanation we shall concentrate on the case of a codimension one algebraic variety $Y$ in complex projective $N$ space, $P^{N}$. The generalization to higher codimension varieties in products of complex projective spaces is straightforward, although technically tedious. We shall discuss some more complicated examples shortly. The calculation relies on the following three exact sequences:

$$
\begin{gathered}
\left.0 \rightarrow E_{P^{N}}(-d) \rightarrow E_{P^{N}} \rightarrow E_{P^{N}}\right|_{Y} \rightarrow 0, \\
0 \rightarrow T_{Y}-\left.\left.T_{P^{N}}\right|_{Y} \rightarrow \mathcal{O}_{P^{N}}(d)\right|_{Y} \rightarrow 0, \\
0 \rightarrow \mathcal{O}_{P^{N}} \rightarrow \mathcal{C}_{P^{N}}(1)^{\oplus N+1} \rightarrow T_{P^{N}} \rightarrow 0,
\end{gathered}
$$

where $E_{P^{N}}$ is a holomorphic vector bundle over $P^{N}, Y$ is a divisor on $P^{N}$ of divisor class $[d], T_{Y}$ represents the tangent bundle of $Y,\left.T_{P^{N}}\right|_{Y}$ represents the tangent bundle 
of $P^{N}$ restricted to $Y$, and $\mathscr{O}_{P^{N}}(d)$ is the tensor product of $d$ copies of the hyperplane bundle. Generally, the notation $A(b)$ denotes $A \otimes \mathcal{O}_{P^{N}}(b)$. Notice that the second exact sequence allows us to relate bundles over $Y$ over the ambient space restricted to $Y$. The first sequence allows us to further relate such bundles over the ambient space restricted to $Y$ to bundles over the ambient space. Now, by use of Bott's formula, Kunneth formula and (2.3) we know the cohomology of any vector bundle over $P^{N}$ (or products of projective spaces) built from tensor products of the tangent bundle with arbitrary line bundles. For later use we reproduce Bott's formula here:

$$
h^{q}\left(P^{N}, \Omega^{p}(k)\right)=\left\{\begin{array}{cc}
\left(\begin{array}{c}
k+N-p \\
k
\end{array}\right)\left(\begin{array}{c}
k-1 \\
p
\end{array}\right) & q=0, k>p \\
1 & k=0, p=q \\
\left(\begin{array}{c}
-k+p \\
-k
\end{array}\right)\left(\begin{array}{c}
-k-1 \\
N-p
\end{array}\right) & q=N, k<p-N \\
0 & \text { otherwise. }
\end{array}\right.
$$

We generally define $h^{q}\left(K, V_{K}\right)$ to be the dimension of $H^{q}\left(K, V_{K}\right)$, where $V_{K}$ is a holomorphic vector bundle over the manifold $K$, and $H^{q}\left(K, V_{K}\right)$ may be considered (by the Dolbeault theorem) as the $q^{\text {th }}$ Dolbeault cohomology group of $M$ taking values in the bundle $V_{K}$. In Bott's formula, $\Omega^{p}$ denotes the $p^{\text {th }}$ antisymmetric power of $T_{P^{N}}^{*}$, the holomorphic cotangent bundle of $P^{N}$. There are two notational conventions which we introduce at this point: first, we shall sometimes omit the subscript in $H^{*}\left(K, V_{K}\right)$ and write $H^{*}(K, V)$ when there is no possibility of confusion, and second, bundles such as $T$ without a subscript refer to the tangent bundle of $Y$, whereas the tangent bundle of the ambient projective space will always carry an explicit subscript, $T_{P^{N}}$. In addition to the dimensions of these cohomology groups over $P^{N}$, we will need one further piece of information: $h^{j}\left(P_{N},\left(T \otimes T^{*}\right)_{P^{N}}\right)=\delta_{0 . j}$. This follows from the fact that $T_{P^{N}} \otimes T_{P^{N}}^{*}=$ End $T_{P^{N}} \oplus \mathcal{C}_{P^{N}}$, and by the rigidity of $P^{N}$. In fact, as we shall mention below, stability of End $T$ implies that $h^{0}\left(Y, T \otimes T^{*}\right)=1$ as well.

Our approach therefore is to relate, via the above sequences, the cohomology of End $T$ to that of bundles over an ambient projective space for which Bott's formula is applicable.

To make this concrete, we now compute $H^{*}\left(Y_{4,5}\right.$, End $\left.T\right)$ for the simplest Calabi-Yau manifold, the quintic hypersurface $Y_{4,5}$ in $P^{4}$. That is, the vanishing locus of a quintic polynomial say,

$$
\sum_{i=1}^{i=5} z_{i}^{5}=0
$$

where $\left(z_{1}, z_{2}, z_{3}, z_{4}, z_{5}\right)$ are homogeneous coordinates for $P^{4}$. For ease of notation we shall often refer to this manifold simply as $Y$. We see that $d=5$ and $N=4$ in (2.1), (2.2), (2.3). We will work with arbitrary $N$ and $d$ for later use. Dualizing sequence (2.2 (i.e. reversing the orientation and dualizing the bundles) and then tensoring the result with $T_{Y}$ (tensoring preserves the exactness), we get

$$
\left.0 \rightarrow T(-d) \rightarrow T \otimes T_{P^{N}}^{*}\right|_{Y} \rightarrow\left(T \otimes T^{*}\right) \rightarrow 0 .
$$


Likewise, tensoring sequence (2.2) with $\left.\mathcal{O}(-d)\right|_{\mathrm{Y}}$, we get

$$
\left.0 \rightarrow T(-d) \rightarrow T_{P^{N}}(-d)\right|_{Y} \rightarrow \mathcal{O}_{Y} \rightarrow 0,
$$

and setting $E=T_{P^{N}}(-d)$ in sequence (2.1),

$$
\left.0 \rightarrow T_{P^{N}}(-2 d) \rightarrow T_{P^{N}}(-d) \rightarrow T_{P^{N}}(-d)\right|_{Y} \rightarrow 0 .
$$

Now tensor (2.2) with $\left.T_{P^{N}}^{*}\right|_{Y}$,

$$
\left.\left.\left.0 \rightarrow T \otimes T_{P^{N}}^{*}\right|_{Y} \rightarrow\left(T \otimes T^{*}\right)_{P^{N}}\right|_{Y} \rightarrow T_{P^{N}}^{*}(d)\right|_{Y} \rightarrow 0,
$$

and tensor (2.3) with $T_{P^{N}}^{*}(-d)$,

$$
0 \rightarrow T_{P^{N}}^{*}(-d) \rightarrow T_{P^{N}}^{*}(-d+1)^{\oplus(N+1)} \rightarrow\left(T \otimes T^{*}\right)_{P^{N}}(-d) \rightarrow 0 .
$$

Finally, let $E=T_{P^{N}}^{*}(d)$ and $\left(T \otimes T^{*}\right)_{P^{N}}$ in (2.1),

$$
\begin{gathered}
\left.0 \rightarrow T_{P^{N}}^{*} \rightarrow T_{P^{N}}^{*}(d) \rightarrow T_{P^{N}}^{*}(d)\right|_{Y} \rightarrow 0, \\
\left.0 \rightarrow\left(T \otimes T^{*}\right)_{P^{N}}(-d) \rightarrow\left(T \otimes T^{*}\right)_{P^{N}} \rightarrow\left(T \otimes T^{*}\right)_{P^{N}}\right|_{Y} \rightarrow 0 .
\end{gathered}
$$

In terms of the dependence of equations, we have

$$
\begin{gathered}
(2.11) \searrow \\
(2.10) \rightarrow(2.12)^{\nearrow}(2.9) \searrow \\
(2.8) \rightarrow(2.7)^{\nearrow^{(2.6)}}
\end{gathered}
$$

So, as an example of the use of the sequences, let's do the first sequence in detail, for $d=5$ and $N=4$ :

\begin{tabular}{l|lrc}
$(2.11)$ & $T_{P^{4}}^{*}$ & $T_{P^{4}}^{*}(5)$ & $\left.T_{P^{4}}^{*}(5)\right|_{Y}$ \\
\hline$h^{0}$ & 0 & 224 & 225 \\
$h^{1}$ & 1 & 0 & 0 \\
$h^{2}$ & 0 & 0 & 0 \\
$h^{3}$ & 0 & 0 & 0 \\
$h^{4}$ & 0 & 0 & 0
\end{tabular}

In this sequence, we have placed the dimensions of the relevant cohomology groups as columns; Bott's formula was used to obtain the first two columns and the third column is calculated using exactness. One proceeds through the chain of exact sequences until reaching (2.6), where we find

\begin{tabular}{l|cll}
$(2.6)$ & $T(-5)$ & $\left.T \otimes T^{* P^{4}}\right|_{Y}$ & $T \otimes T^{*}$ \\
\hline $\mathrm{h}^{0}$ & 0 & $x$ & $y$ \\
$h^{1}$ & 1 & $x+224$ & $y+223$ \\
$h^{2}$ & 0 & 0 & $z+223$ \\
$h^{3}$ & 224 & 1 & $z$
\end{tabular}


where $x$ is equal to 0 or 1 and $y=z$ by Kodaira-Serre duality. In fact, since $T$ is a stable bundle, we know that End $T$ is stable and hence has no global sections. Thus, $y=1$, and so

$$
h^{1}(Y, \text { End } T)=224 .
$$

[Note that, since $h^{1,0}=0$ for $K$ Calabi-Yau, $H^{1}(K$, End $T) \cong H^{1}\left(K, T \otimes T^{*}\right)$.]

\section{Generalizations}

To extend this technique to the case of algebraic varieties in products of complex projective spaces, takes very little in the way of new ideas. We merely need to extend our original sheaf sequences to take account of the "increased" ambient space. For example, if $X \equiv P^{2} \times P^{2}$, and $W$ is the vanishing locus of a polynomial of bidegree $(3,3)$ in $X,(2.1),(2.2),(2.3)$ would now be

$$
\begin{aligned}
0 & \left.\rightarrow E_{X}(-3,-3) \rightarrow E_{X} \rightarrow E_{X}\right|_{W} \rightarrow 0, \\
0 & \left.\rightarrow T_{W} \rightarrow T_{X}\right|_{W} \rightarrow \mathcal{C}_{W}(3,3) \rightarrow 0, \\
0 \rightarrow \mathcal{O}_{X}(-1,-1)^{\oplus 2} & \rightarrow \mathcal{O}_{X}(-1,0)^{\oplus 3} \oplus \mathcal{O}_{X}(0,-1)^{\oplus 3} \rightarrow T_{X}(-1,-1) \rightarrow 0 .
\end{aligned}
$$

Proceeding in the same way as was done in detail for the quintic hypersurface, one finds that $H^{1}(W$, End $T)$ has dimension 176.

One can also carry out this computational procedure for higher codimension varieties. For example, the Calabi-Yau manifold $Y_{5 ; 3,3}$ (the vanishing locus of two homogeneous cubis in $\left.C P^{5}\right)$ has $h^{1}\left(Y_{5 ; 3,3}\right.$, End $\left.T\right)=140$, and it is not difficult to generate these dimensions for the other complete intersection Calabi-Yau manifolds which are embedded in one projective space.

We should mention that to this point, we have only made use of the most superficial information provided by the exact sequences: namely the relationship between the dimensions of the cohomology groups which appear. Much more information is contained in the maps which realize these sequences. For some manifolds, the computation of the dimension of $H^{1}(K$, End $T)$ requires the information embodied in these maps. A example is that of the only three generation complete intersection Calabi-Yau manifold: the vanishing locus, in $P^{3} \times P^{3}$ of bi-degree $(3,0),(0,3)$, and $(1,1)$ homogeneous polynomials [11]. If one follows the above algorithm in this case, one finds that there are not enough vanishing dimensions in the long exact cohomology sequences to determine the number of $E_{6}$ singlets simply from a study of the dimensions of the surrounding cohomology groups.

By studying the maps which realize the sequences involved in this computational strategy, this ambiguous conclusion could probably be rectified.

\section{Deformation Theory}

Until now we have concentrated on the first method of computing cohomology that we mentioned earlier, namely exact sequences. We now discuss the second: deformation theory. 
It is important to bear in mind that elements in $H^{1}(K$, End $T)$, as emphasized in this context in [12] have a geometric interpretation. They represent deformations to the complex structure of the tangent bundle $T_{K}$. By counting the number of independent deformations to the complex structure of the tangent bundle, then, one should be able to determine the dimension of $H^{1}(K$, End $T)$. To this end, in [12] the following algorithm is introduced:

First note that a tangent vector ${ }^{1}$ to $P^{N}$ is equivalent to a collection of $n$ linear functions $\left\{V^{a}\right\}$ subject to the projectivity constraint

$$
V^{a}=V^{a}+\lambda z^{a},
$$

where the $\left\{z^{a}\right\}$ are homogeneous $P^{N}$ coordinates and $\lambda$ is a complex number. For this $P^{N}$ tangent vector field to be tangent to the hypersurface defined by the vanishing of a homogeneous polynomial function $P\left(z^{a}\right)$ the $V^{a}$ must further satisfy

$$
V^{a} P_{a}=0
$$

where $P_{a}=\partial P / \partial z^{a}$. Having thus essentially defined the tangent bundle to our hypersurface, we can now ask about ways in which we can holomorphically deform it. The answer to this question lies in relaxing (4.2) to

$$
V^{a} Q_{a}=0,
$$

where the $Q_{a}$ are any homogeneous polynomials whose order is the same as that of the $P_{a}$. The latter, as shown in [12] are in one to one correspondence with tensors of the form $p_{a_{1}, a_{2}, \ldots, a_{n}}$ which are completely symmetric on the last $n-1$ indices, and vanish when symmetrized on all $n$ indices. Thus, in this computational procedure, the calculation of $H^{1}(Y$, End $T)$ is reduced to a simple combinatoric exercise. For example, on $Y_{4,5}$ one easily computes that the dimension of the space of such tensors is 224, in agreement with the exact sequence calculation above.

Of primary importance is to understand the conditions under which this procedure correctly counts the dimension of $H^{1}(Y \text {, End } T)^{2}$. To shed some light on this question it is useful to rephrase the above deformation algorithm in a language more suited to our exact sequence calculation. We will illustrate this, again, in the simple case of $Y_{4,5}$.

The tensor $p_{a b c d e}$ being symmetric on its last four indices and vanishing upon complete symmetrization of all five indices may be thought of as an element of the kernel of the map

$$
\phi: H^{0}\left(P^{4}, O^{\oplus 5}(4)\right) \rightarrow H^{0}\left(P^{4}, O(5)\right) .
$$

If one dualizes the sequence (2.3) and tensors it with $\mathcal{O}_{p^{4}}(5)$ one sees that this kernel is isomorphic to

$$
H^{0}\left(P^{4}, T_{P^{4}}^{*}(5)\right)
$$

\footnotetext{
${ }^{1}$ For simplicity of notation we describe this algorithm for one polynomial constraint in one projective space

${ }^{2}$ The related question of the efficacy of deformation theory in computing $h^{1}(K, T)$ has been answered in [10]
} 
We thus see that the deformation method, in this case, is computing the dimension of the latter cohomology group. Notice that the cohomology of the bundle $T_{P^{4}}^{*}(5)$ enters into the calculation of $H^{1}\left(Y_{4,5}\right.$, End $\left.T\right)$ through sequence (2.11). In fact, with not much effort, one can see from the precise structure of cohomology sequences associated with the exact sequences (2.11), (2.9), (2.6) that

$$
H^{0}\left(P^{4}, T_{P^{4}}^{*}(5)\right) \cong H^{1}\left(Y_{4,5}, \text { End } T\right),
$$

and hence, the polynomial deformation algorithm is justified in this case. Now, this is as expected since we already mentioned that the deformation procedure on $Y_{4,5}$ yields $224 E_{6}$ singlets. The latter justification from exact sequences is useful, however, in that we see that the argument requires a number of special cohomological features. Among these requirements are $h^{2}\left(Y_{4.5}, T(-5)\right)=0$ and $h^{1}\left(Y_{4,5},\left(T \otimes T^{*}\right)_{\left.P^{4}\right|_{Y_{4}, 5}}\right)=0$. In the case of $K 3$ (the quartic surface in $\left.P^{3}\right)$ the condition analogous to the first of these requirements is not met, and we find that $h^{1}(K 3$, End $T)=90$ [this can be computed using the exact sequences (2.6)-(2.12) with $d=4$ and $N=3$, or more easily, by using index theory] while the deformation algorithm only yields 45 [which from our discussion we know to be $h^{0}\left(P^{3}, T_{P^{3}}^{*}(4)\right)$ ]. For the case of the bicubic equation in $X=P^{2} \times P^{2}$, the second of these conditions is not met. Our exact sequence calculation showed $h^{1}(W$, End $T)=176$, while the deformation method yields 160 . It is easy to see that the discrepancy of 16 comes precisely from the 16 elements in $H^{1}\left(X\right.$, End $\left.T_{X}\right)$.

In conclusion, we have presented a computational strategy for determining endomorphism valued cohomology, an essential ingredient for determining the low energy singlet spectrum of compactified superstring models. We have applied our method to a number of examples, and compared it with the polynomial deformation method. We have shown that only under highly favourable circumstances does the polynomial deformation method correctly compute the desired cohomology, and we have indicated the obstructions in a coupled of examples. As for the phenomenologically interesting case of the three generation manifold, our procedure can probably be pushed further to accomplish this task, but in the form presented in this paper, it simply is not sufficiently robust. We hope to return to this question in a future publication.

Note added in proof. We are aware that P. Green, T. Hubsch, and A. Lutken have computed $h^{1}($ End $T)$ for certain Calabi-Yau manifolds [13] and their results agree with those presented herc, and with other cxamples we have not included.

\section{References}

1. Gross, D., Harvey, J., Martinec, E., Rohm, R.: Nucl. Phys. B 256, 75 (1985)

2. Dixon, L., Jarvey, J., Vafa, C., Witten, E.: Nucl. Phys. B 261, 678 (1985)

3. Candelas, P., Horowitz, G., Strominger, A., Witten, E.: Nucl. Phys. B 258, 75 (1985)

4. Witten, E.: Nucl. Phys. B 268, 79 (1986)

5. For a review see Greene, B.R., Kirklin, K.H., Miron, P.J.: Topology and geometry in superstring inspired phenomenology. In: Mathematical aspects of string theory. Yau, S.-T. (ed.). Vol. I: 441 (1987)

6. Greene, B.R., Kirklin, K.H., Miron, P.J., Ross, G.G.: Nucl. Phys. B 278, 667 (1986) 
7. Hübsch, T.: Commun. Math. Phys. 108, 291 (1987); Green, P., Hübsch, T.: Commun. Math. Phys. 109, 99 (1987)

8. Green, P., Hübsch, T., Lutken, C.: Univ. of Maryland preprint, 1988

9. Witten, E.: Nucl. Phys. B 258, 75 (1985)

10. Hübsch, T., Green, P.: Commun. Math. Phys. 113, 505 (1987)

11. Yau, S.-T.: Argonne symposium on anomalies, geometry and topology. Singapore: World Scientific 1985

12. Green, M.B., Schwarz, J.H., Witten, E.: Superstring theory, Vol. II. Cambridge: Cambridge University Press 1987

13. Hübsch, T.: Private communication

Communicated by S.-T. Yau

Received July 15, 1988; in revised form September 10, 1988 